Heron Mendez-Sanchez, Wilbert Mendez-Vivas, Gary Kosai Vargas-Mendoza, Saul Vazquez-Lopez, Angela Dayana Williams-Jacquez, Arturo Cortes-Telles

Hospital Regional de Alta Especialidad de la Península de Yucatán, Yucatán, México

\title{
Solitary fibrous tumors of the pleura: a clinical-pathological characterization emphasizing changes in lung function
}

\begin{abstract}
Solitary fibrous tumors of the pleura (SFTP) represent less than $5 \%$ of all pleural tumors with a higher susceptibility for men in the sixth and seventh decades of life. Imaging and histopathological analyses indicate that these tumors have an unpredictable clinical course since larger tumors are often associated with malignancy; however, this assumption is not universal. In addition, there are no systematic descriptions of lung function in either the basal or post-surgical scenarios of SFTP patients. We describe a series of 3 patients between $4^{\text {th }}$ and $5^{\text {th }}$ decade of life with a benign histopathological SFTP without local recurrence in their follow-up. We also analyzed changes in lung function, emphasizing possible associations with clinical recovery.
\end{abstract}

Key words: solitary fibrous tumor of the pleura, pleural tumors, pathology, lung function

Adv Respir Med. 2019; 87: 247-251

\section{Introduction}

Since the initial categorization of solitary fibrous tumors of the pleura (SFTP) [1], the literature worldwide contains descriptions of over 800 cases, highlighting clinical and histopathological aspects, tumor dimensions and trans-operatory eventualities. Currently, it is estimated that SFTP has an incidence rate of 2.8 cases per 100,000 registered hospital patients [2]. Solitary fibrous tumors of the pleura can affect individuals of all ages; however, it is most often reported in the sixth or seventh decade of life. Men and women are equally affected without relation to race. Clinically, the range of expression varies from asymptomatic (incidental findings) to such frequent manifestations as thoracic pain, coughing and dyspnea [3-4]. We found no descriptions of lung function to determine to what degree those changes are representative of the disease's clinical impact [5]. Accordingly, we sought to characterize the clinical and pathological behavior of the population treated at our hospital in the last five years, emphasizing lung function changes.

\section{Case 1}

A 37-year-old female with a history of well-controlled asthma and otherwise healthy, without smoking, use of biomass fuels, asbestos or other dust exposures sought medical attention after 2 years of progressive moderate-to-severe dyspnea (MRC 3). She also complained about malaise, a sensation of fullness in her right hemithorax, without weight loss. On physical examination, dulled percussion was found in the right hemithorax. A computed tomography (CT) scan of the chest revealed a large-volume tumor adhered to the visceral and parietal pleura (Figure 1A). Pre-surgical spirometry revealed an FVC of $0.89 \mathrm{~L}(30 \%)$.

Once informed consent was signed, surgery was performed to resect the tumor that measured $20 \times 25 \mathrm{~cm}$. Total blood loss during surgery was 2000 cc. The pathology report confirmed an SFTP and less than one mitosis per high-power field (HPF) (Figure 2A). During follow-up

\footnotetext{
Address for correspondence: Arturo Cortes-Telles, Departamento de Neumología y Cirugía de Tórax, Hospital Regional de Alta Especialidad de la Península de Yucatán, Yucatán, México; e-mail:dr_morenheim@hotmail.com

DOI: 10.5603/ARM.a2019.0042

Received: 25.03 .2019

Copyright (C) 2019 PTChP

ISSN 2451-4934
} 

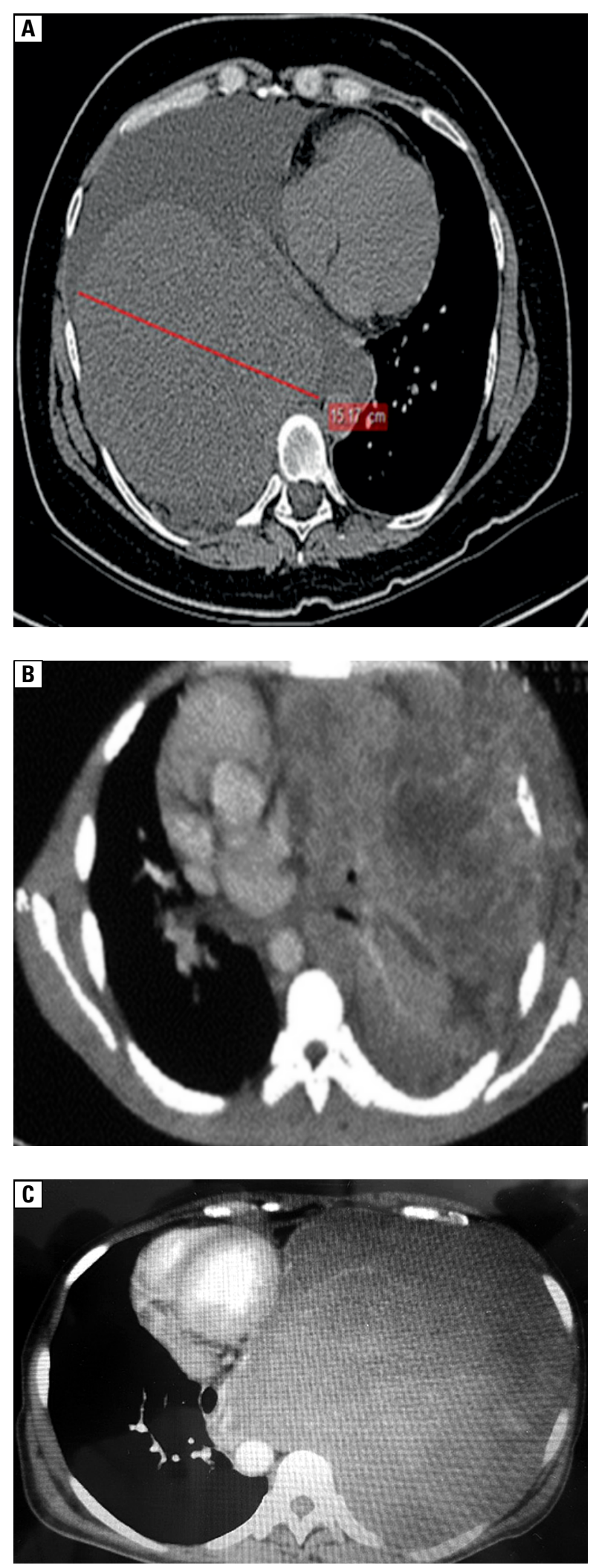

Figure 1. A. Axial chest CT scan showed right pleural tumor involving middle and inferior lobes. B. Axial chest CT scan showed a large heterogeneous tumor in left hemithorax with displacement of mediastinal vascular structures. C. Axial chest CT scan, showed a large mass with significant displacement of mediastinal structures
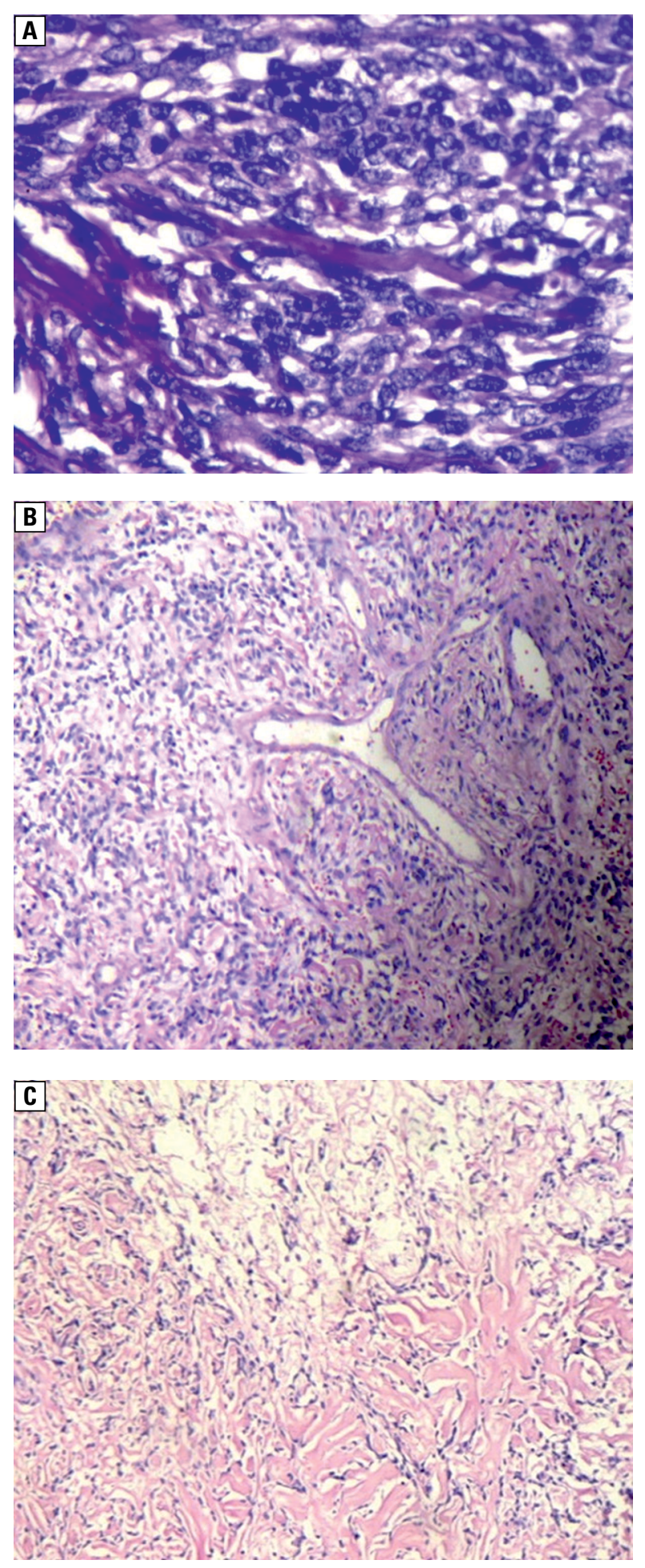

Figure 2. A. Fusiform cells mixed with collagen bundles $(40 \times)$. B. Vascular pattern on deer antlers $(10 \times)$. C. Collagen bundles with myxoid appearance areas $(10 \times)$

(6 months), lung function (Table 1) showed significant improvement of FVC $(2.83 \mathrm{~L}, 93 \%$ predicted). After 21 months of surgery, the patient is respiratory asymptomatic and without signs of disease recurrence. 
Table 1. Differences of baseline and postoperative pulmonary function

\begin{tabular}{|c|c|c|c|}
\hline Variable & Baseline & Postoperative & $\begin{array}{c}\text { Change } \\
\text { (postoperative-baseline) }\end{array}$ \\
\hline \multicolumn{4}{|l|}{ Case 1} \\
\hline FVC (\%p) & 30 & 93 & $218 \%$ \\
\hline FVC (Lts) & 0.89 & 2.83 & 1.94 Lts \\
\hline $\mathrm{FEV}_{1}(\% \mathrm{p})$ & 39 & 93 & $222 \%$ \\
\hline $\mathrm{FEV}_{1}$ (Lts) & 0.73 & 2.35 & 1.62 Lts \\
\hline $\mathrm{FEV}_{1} / \mathrm{FVC}(\%)$ & 83 & 83 & \\
\hline \multicolumn{4}{|l|}{ Case 2} \\
\hline FVC (\%p) & 38 & 71 & $87 \%$ \\
\hline FVC (Lts) & 1.57 & 3.02 & 1.45 Lts. \\
\hline $\mathrm{FEV}_{1}(\% \mathrm{p})$ & 40 & 81 & $103 \%$ \\
\hline $\mathrm{FEV}_{1}$ (Lts) & 1.32 & 2.73 & $1.41 \mathrm{Lts}$ \\
\hline $\mathrm{FEV}_{1} / \mathrm{FVC}(\%)$ & 84 & 90 & \\
\hline \multicolumn{4}{|l|}{ Case 3} \\
\hline FVC (\%p) & 24 & 67 & $179 \%$ \\
\hline FVC (Lts) & 0.68 & 1.92 & 1.24 Lts \\
\hline $\mathrm{FEV}_{1}(\% \mathrm{p})$ & 22 & 69 & $214 \%$ \\
\hline $\mathrm{FEV}_{1}$ (Lts) & 0.53 & 1.62 & $1.09 \mathrm{Lts}$ \\
\hline $\mathrm{FEV}_{1} / \mathrm{FVC}(\%)$ & 78 & 84 & \\
\hline
\end{tabular}

$\mathrm{FVC}$ - forced vital capacity; $\mathrm{FEV}_{1}$ — forced expiratory volume in 1st second of expiration

\section{Case 2}

A 43-year-old male without smoking or exposure to dust or biomass fuels sought medical attention after a year of progressive mild-to-moderate dyspnea (MRC 2). He also complained about intermittent oppressive chest pain. The physical examination revealed dulled percussion in the left hemithorax, otherwise normal. The chest CT scan showed a highly vascularized tumor measuring $13 \times 18 \mathrm{~cm}$ (Figure 1B). Baseline lung function showed an FVC of $1.57 \mathrm{~L}$ (38\% predicted).

A biopsy was taken following written informed consent due to the vascular findings on CT. The pathology analysis reported the presence of vascularized mesenchymal neoplasia without atypia. Surgery was performed to resect the tumor. The size was $15 \times 15 \mathrm{~cm}$ and total blood loss was 800 cc. Pathology confirmed an SFTP with less than 2 mitoses per HPF (Figure 2B). After 6 months, spirometry testing showed an increase in FVC to $3.02 \mathrm{~L}$ ( $71 \%$ predicted). After 11 months of surgery, the patient is alive without recurrence.

\section{Case 3}

A 37-year-old otherwise healthy female sought medical attention after 3 years with pro- gressive dyspnea (MRC) accompanied by weight loss (13 kg) and intermittent fever. The chest CT scan confirmed a tumor in the left hemithorax that displaced the mediastinal structures and pleural effusion (Figure 1C).

Baseline spirometry showed an FVC of $0.68 \mathrm{~L}$ (24\%). After informed consent was signed, surgery was performed and a tumor of $45 \times 35 \mathrm{~cm}$ was resected from the left hemithorax with a total blood loss of 1200 cc (Figure 2C). Pathology confirmed the existence of SFTP without evidence of malignancy and less than 1 mitosis per HPF.

During follow-up, FVC increased to $1.92 \mathrm{~L}$ (67\%). Currently; the patient has a disease-free survival of 7 months with no recurrence.

\section{Discussion}

The findings considered to be relevant in these clinical cases are as follows: a) age at the time of diagnosis; b) the disease-free survival; c) the size of the SFTP; and d) the improvement in lung function.

Despite SFTP being more prevalent between $6^{\text {th }}$ and $7^{\text {th }}$ decade of life, it can affect young people. Indeed, previous reports have described 
a range of ages between 34 and 83 years [6]. In a Hispanic case-series, we have demonstrated that around $20 \%$ of patients are under 50 years of age at the time of diagnosis [3-4]. Virtually all published reports do not evaluate possible hypotheses concerning the age at diagnosis or gender predominance.

At the time of writing the present manuscript, our cases showed a mean disease-free survival of 13 months without signs of recurrence or malignancy. Previous reports have estimated a disease-free survival in a Hispanic population up to 48.5 months [3]. There is scarce information regarding surveillance after resection in patients with SFTP. Tapias et al., developed a scoring system based on pathology findings: a) parietal pleura origin; b) sessile morphology; c) size of tumor $>10 \mathrm{~cm}$; d) presence of hypercellularity; e) necrosis and mitotic activity ( $>4 / \mathrm{HPF}$ ). Recurrence free-survival was $100 \%$ up to 15 years with a score $<3$ [7]. In addition, You et al. have used CT scan findings to differentiate benign from malignant SFTP, showing that, in contrast to benign tumors, malignant tumors: a) were greater in size; b) had a higher percentage rate of inhomogeneous density; c) had increased tumor vascularity; and d) showed pleural effusions. Based on these reports, it is suggested that surveillance should be considered up to 24 months, accompanied by imaging evaluations - CT scans - every six months [8, 9].

Regarding the size, most cases of SFTP are described as tumors $<10 \mathrm{~cm}$ incidentally discovered in asymptomatic patients during medical evaluations that include a chest x-ray [10]. It is thought that the larger the tumor the higher the risk of malignancy [7]. Nonetheless, Harrison et al. reported a mean size for malignant SFTP of $12 \mathrm{~cm}$ [6]. The dimensions of the SFTP presented herein are not the ones commonly reported previously, and none were associated with malignancy. We can conclude that SFTP might have "an unpredictable" behavior if clinicians consider only the size. SFTP larger than $18 \mathrm{~cm}$, or that occupy at least $40 \%$ of the total volume of a hemithorax, are referred to as "Giant SFTP". They represent a challenge for surgical resection due to less anatomical exposure, greater risk of blood loss, and significant adherences [11]. To the best of our knowledge, in Mexico and Latin America, there is only one case report on SFTP that have dimensions up to $40 \mathrm{~cm}$ [4]. One of our cases is, to our knowledge, the largest tumor resected $(45 \mathrm{~cm})$ and showed benign behavior without vascular complications.

Finally, regarding lung function in SFTP patients, we were unable to find any detailed analy- sis or reliable description to compare our findings. However, we did explore possible explanations that could have effects after surgical resection of SFTP. Whenever a tumor occupies a volume in the affected hemithorax, respiratory mechanics and lung compliance become compromised. For example, an increase in SFTP volume decreases lung volume, resulting in a rise in airway resistance. These physiological changes ultimately increase the driving pressure needed to generate a given tidal volume. This can be compensated through an augmented inspiratory muscle effort and alterations in breathing patterns (rapid shallow breathing), but this likely comes at the expense of increased dyspnea.

Abnormalities in the pleural and intrathoracic pressure become relevant as the main components of the transmural pressure, which has an important influence on many aspects of pulmonary function. Once the tumor volume increases, the transmural pressure gradient decreases, compromising ventilation/perfusion ratios and therefore gas exchange, another physio-pathological explanation of dyspnea [12].

As this condition worsens, accessory muscles of inspiration are activated generating a more pronounced decrease in the intrapleural and alveolar pressure that enables the movement of airflow. As a consequence, energy expenditure increases, triggering the appearance of symptoms such as chest pain and fatigue [13].

Regarding lung function, baseline measurements in our patients suggest a severe restrictive ventilatory pattern (FVC below $40 \%$ predicted); however, during post-surgical surveillance of at least 6 months, we observed a tremendous functional recovery showing a mild-to-moderate restrictive pattern (FVC above $60 \%$ predicted). Numerically, the absolute change was two-fold higher than baseline measurements. Collectively, these changes can be explained by the post-surgical improvements in compliance of the pulmonary tissue, thoracic cage, and airway resistance, given that pulmonary volume is compromised proportionally to tumor size [12, 13]. We acknowledge that total lung capacity was not measured in our patients in order to confirm a restrictive ventilatory pattern. If available, plethysmography would provide additional insight into changes in pulmonary function following surgery. However, in contrast to spirometry, plethysmography is not always available in routine clinical practice.

Based on the current analysis, we consider it extremely important to continue the follow-up of SFTP patients by measuring pulmonary 
function and performing CT scans, as these two approaches will provide an objective evaluation of patients' functional and structural changes following surgery.

\section{Conclusion}

SFTP are rare pleural neoplasms with a final diagnosis being obtained after surgical resection. Lung function should be routinely measured before surgery since we have shown that these patients can have a restrictive ventilatory pattern that improves after surgery.

\section{Conflict of interest}

The authors declare no conflict of interest.

\section{References:}

1. Chick JF, Chauhan NR, Madan R. Solitary fibrous tumors of the thorax: nomenclature, epidemiology, radiologic and pathologic findings, differential diagnoses, and management. AJR Am J Roentgenol. 2013; 200(3): W238-W248, doi: 10.2214/ AJR.11.8430, indexed in Pubmed: 23436868.

2. Cardillo G, Lococo F, Carleo F, et al. Solitary fibrous tumors of the pleura. Curr Opin Pulm Med. 2012; 18(4): 339-346, doi: 10.1097/ MCP.0b013e328352f696, indexed in Pubmed: 22450304.

3. Fuentes-Valdés E, Martín-González MA. Tumor fibroso solitario de la pleura. Descripción clínico quirúrgica de 10 casos. Neumol Cir Torax. 2014; 73(3): 174-178.

4. Pinedo-Onofre J, Robles-Pérez E, Peña-Mirabal E, et al. Tumor fibroso solitario gigante de la pleura. Cir Ciruj. 2010; 78: 31-43.
5. Mohamed H, Mandal AK. Natural history of multifocal solitary fibrous tumors of the pleura: a 25-year follow-up report. J Natl Med Assoc. 2004; 96(5): 659-662, indexed in Pubmed: 15160981.

6. Harrison-Phipps KM, Nichols FC, Schleck CD, et al. Solitary fibrous tumors of the pleura: results of surgical treatment and long-term prognosis. J Thorac Cardiovasc Surg. 2009; 138(1): 19-25, doi: 10.1016/j.jtcvs.2009.01.026, indexed in Pubmed: 19577049.

7. Tapias LF, Mino-Kenudson M, Lee H, et al. Risk factor analysis for the recurrence of resected solitary fibrous tumours of the pleura: a 33-year experience and proposal for a scoring system. Eur J Cardiothorac Surg. 2013; 44(1): 111-117, doi: 10.1093/ ejcts/ezs629, indexed in Pubmed: 23233072.

8. You X, Sun X, Yang C, et al. CT diagnosis and differentiation of benign and malignant varieties of solitary fibrous tumor of the pleura. Medicine (Baltimore). 2017; 96(49): e9058, doi: 10.1097/MD.0000000000009058, indexed in Pubmed: 29245313.

9. Gupta A, Souza CA, Sekhon HS, et al. Solitary fibrous tumour of pleura: CT differentiation of benign and malignant types. Clin Radiol. 2017; 72(9): 796.e9-796.e17, doi: 10.1016/j. crad.2017.03.028, indexed in Pubmed: 28495011.

10. Cardinale L, Ardissone F, Garetto I, et al. Imaging of benign solitary fibrous tumor of the pleura: a pictorial essay. Rare Tumors. 2010; 2(1): e1, doi: 10.4081/rt.2010.e1, indexed in Pubmed: 21139938.

11. Cardillo G, Carbone L, Carleo F, et al. Solitary fibrous tumors of the pleura: an analysis of 110 patients treated in a single institution. Ann Thorac Surg. 2009; 88(5): 1632-1637, doi: 10.1016/j.athoracsur.2009.07.026, indexed in Pubmed: 19853123.

12. Lutfi MF. The physiological basis and clinical significance of lung volume measurements. Multidiscip Respir Med. 2017; 12: 3, doi: 10.1186/s40248-017-0084-5, indexed in Pubmed: 28194273.

13. Flesch JD, Dine CJ. Lung volumes: measurement, clinical use, and coding. Chest. 2012; 142(2): 506-510, doi: 10.1378/ chest.11-2964, indexed in Pubmed: 22871760. 